\title{
Operational Amplifier based Schmitt Trigger with Digitally Controllable Hysteresis
}

\author{
Faroze Ahmad \\ Department of Electronics and Communication Engineering \\ Islamic University of Science and Technology (IUST) \\ Awantipora-192122, Pulwama India
}

\begin{abstract}
A simple and low-cost circuit for controlling hysteresis of Schmitt trigger is proposed in this paper. The hysteresis of the circuit is digitally tuned by employing binary-weighted resistors in place of feedback resistor. The behavior of proposed circuit has been tested experimentally in hardware. The results obtainedare satisfactory and are also presented.
\end{abstract}

\section{Keywords}

Binary Weighted Resistors, Operational Amplifier, Schmitt trigger.

\section{INTRODUCTION}

Schmitt triggers are widely used for enhancing noise immunity in various circuits. They find applications in medical instruments, sub-threshold SRAM, buffer, image sensors and pulse width modulation applications [1]-[2]. The Schmitt trigger with adjustable hysteresis can be used for different environments with different noise levels. Therefore the demand for variable hysteresis of Schmitt trigger has increased in many systems [3]. Several circuits with variable hysteresis using few components, offering high speed and high accuracy, have been reported in the literature, but are not digitally tunable [1], [4]-[5]. There are situations where accuracy and high speed are not only considerations but their compatibility with digital systems is essential. Fei introduced a high speed adjustable hysteresis circuit but the curcuit is not digitally programmable [1].The circuits proposed with digitally tunable hysteresis are complex. Jiri Misurec et al proposed a circuit with digitally programmable hysteresis [6]. The circuit employs current conveyors, operational amplifiers and $\mathrm{A} / \mathrm{D}$ converter to achieve digitally controllable hysteresis. Though the circuit offers high speed and accuracy, but can't prove economical keeping in view the hardware requirement.In 2001 Prodanov et alpresented a circuit,using two operational amplifiers, Logic gates and Mosfet-OnlyCurrent-Divider(MOCD) [7]. The circuit is compatible with VLSI fabrication but requires more hardware.There are situations where simplicity and low cost of circuits are desirable. In this paper a very simple and low-cost circuit with digitally variable hysteresis is proposed. The circuit uses only one operational amplifier and a few components. The remaining part of this paper is organized as follows. In Section 2, the back ground of schmitt trigger is discussed. Section 3 discusses the proposed circuit with programmable hysteresis. Finally in Sections 4 and 5 experimental results and conclusion are respectively presented.

\section{TRADITIONAL SCHMITT TRIGGER}

A Schmitt trigger is basically constructed by using operational amplifier with positive feedback. In a Schmitt trigger circuit the output changes when the input signal coincide a certainvoltage level known as threshold voltage [8]. A simple schmitt trigger circuit using operational amplifier is shown in Fig. 1 (a).

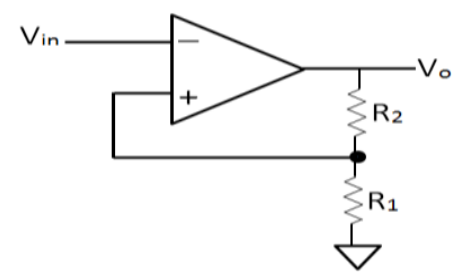

Fig 1(a):A Non-programmable Schmitt trigger

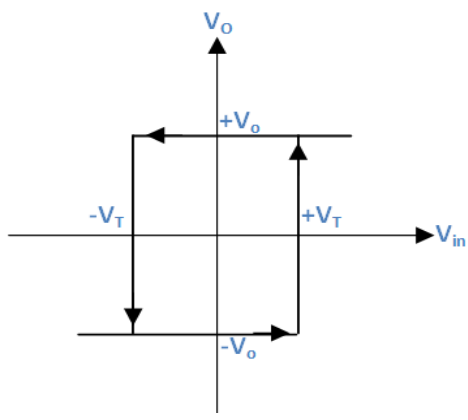

Fig.1(b ): Hysteresis Curve

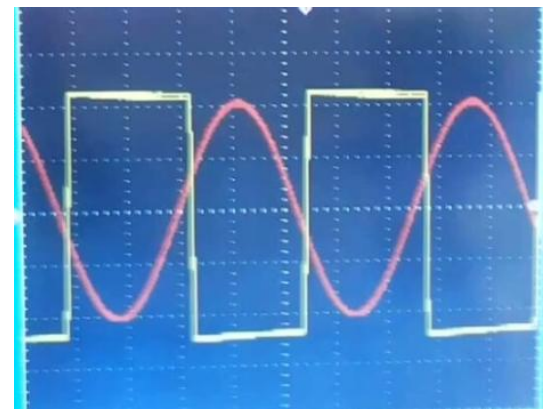

Fig 1(c): Waveform of Traditional Schmitt trigger

The output of this circuit switches from one saturation to another when the input signal coincides with the threshold viltage $\mathrm{V}_{\mathrm{T}}$ as shown in hysteresis curve (Fig. 1(b)). The behaviour of output with respect to input signal, as experimented out in Laboratory, is also shown in Fig. 1(c). It can be seen from the waveform that whenever the input sinusoidal signal reaches a certain level, the output swings from positive to negative saturation.

The threshold voltage $\left(\mathrm{V}_{\mathrm{T}}\right)$ is known as Upper threshold $\left(\mathrm{V}_{\mathrm{ut}}\right)$ and Lower threshold $\left(\mathrm{V}_{\mathrm{lt}}\right)$ respectively for positive and negative output $\left(\mathrm{V}_{\mathrm{o}}\right)$ of the schmitt trigger. Consider $\mathrm{V}_{\mathrm{o}}$ is positive, threshold voltage is $+\mathrm{V}_{\mathrm{T}}$ and is given by

$$
\mathrm{V}_{\mathrm{ut}}=\beta\left(+\mathrm{V}_{\mathrm{o}}\right)
$$


where $\quad \beta=\mathrm{R}_{1} /\left(\mathrm{R}_{1}+\mathrm{R}_{2}\right)$

Now when the input signal slightly becomes greater than $\mathrm{V}_{\mathrm{ut}}$, the output switches from $+\mathrm{V}_{\mathrm{o}}$ to $-\mathrm{V}_{\mathrm{o}}$ and the threshold voltge becomes $-\mathrm{V}_{\mathrm{T}}$ and is givenby

$$
\mathrm{V}_{\mathrm{lt}}=\beta\left(-\mathrm{V}_{\mathrm{o}}\right)
$$

The hysteresis voltage $\left(\mathrm{V}_{\text {hy }}\right)$ is given by

$$
\mathrm{V}_{\mathrm{hy}}=2 \beta\left(\mathrm{V}_{\mathrm{o}}\right)
$$

Thus the hysteresis of a schimtt trigger depends upon its $\beta$. In the proposed circuit the $\beta$ of the circuit is varied by a binary code as discussed in the next section.

\section{PROPOSED CIRCUIT}

Fig.2(a) shows the proposed circuit for digitally programmable Schmitt trigger, wherein eight different hysteresis voltages are achieved. It is a modification of circuit shown in Fig.1 (a) with $\mathrm{R}_{1}$ replaced with a resistor array. The resistors have different range of values which are chosen according to the requirement of threshold voltages. Any one of the resistors $\mathrm{R}_{1 \mathrm{a}}$ through $\mathrm{R}_{1 \mathrm{~h}}$ can be connected to ground by turning on a particular FET switch connected in series with the resistors.

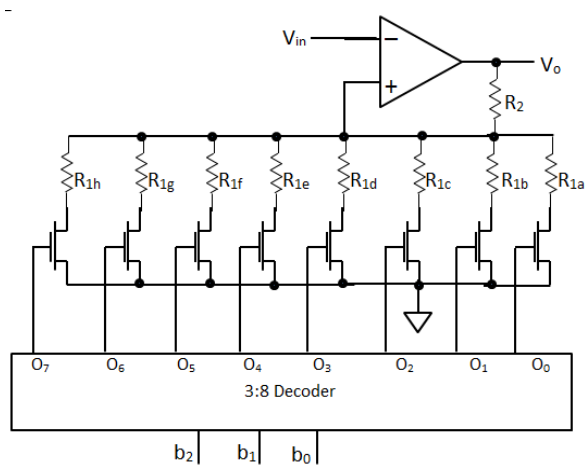

Fig.2(a): Proposed digitally tunable Schmitt trigger with resistor array feedback arrangement

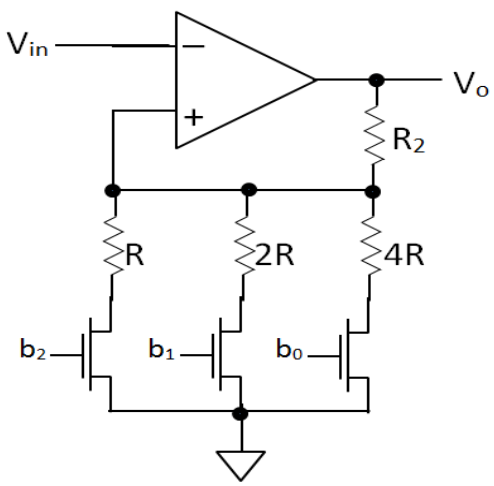

Fig.2(b): Proposed digitally tunable Schmitt trigger with binary weighted resistors feedback arrangement

When a particular binary code is applied to the input bits $b_{2} b_{1} b_{0}$, the corresponding output of the decoder goes high thus turning on one of the FETs, which in turn connects only one of the resistors to ground. Thus the hysteresis voltage levels achieved is equal to $2^{\mathrm{n}}$, where $\mathrm{n}$ is the binary code length. It can be seen that the number of resistors and FETs required is also $2^{\mathrm{n}}$. In order to reduce the hardware complexity and achieve almost same number of hysteresis levels, the resistor array is replaced by a binary weighted resistor network whereas resistor $R_{2}$ is fixed, as shown in Fig. 2(b). Let $R_{e}$ be the effective resistance achieved by the different binary numbers applied to binary weighted network,then $\beta$ of the circuit is given

$\beta=\mathrm{R}_{\mathrm{e}} /\left(\mathrm{R}_{\mathrm{e}}+\mathrm{R}_{2}\right)(4)$

The effective resistance can be calculated by

$\mathrm{R}_{\mathrm{e}}=1 /\left[\left(\mathrm{b}_{2} / \mathrm{R}\right)+\left(\mathrm{b}_{1} / 2 \mathrm{R}\right)+\left(\mathrm{b}_{0} / 4 \mathrm{R}\right)\right](5)$

where $\mathrm{b}_{i}=0 \quad \mathrm{OFF}$

and $\mathrm{b}_{i}=1 \quad \mathrm{ON}$

When all the FET switches are off i.e., $b_{0}=0, b_{1}=0$ and $b_{2}=0$, the total resistance $R_{e}$ becomes infinity. Therefore, binary word 000 is avoided and number of hysteresis voltage levels achieved is $2^{\mathrm{n}-1}$.

\section{EXPERIMENTAL RESULTS}

The workability of the circuit was tested in hardware using operational amplifier IC741. The binary weighted network was implemented by series parallel combination of $4.7 \mathrm{~K}$ resistors, i.e., $2.35 \mathrm{~K}, 4.7 \mathrm{~K}$ and $9.4 \mathrm{~K}$. Three different hysteresis levels were tested at different code words are shown in Fig.3. In Fig 3(a) the binary code applied to binary inputs $b_{2} b_{1} b_{0}$, is 111 . In 3 (b) the code is 001 and in 3(c) 100 . The threshold voltage levels are shown by dashed lines on the waveform. It is clear that $\mathrm{V}_{\mathrm{o}}$ changes wherever the $\mathrm{V}_{\text {in }}$ coincides these dashed lines.

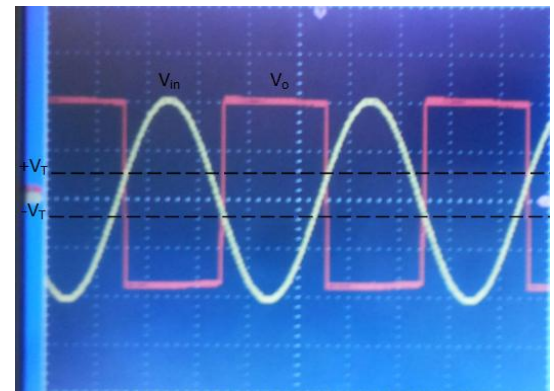

Fig. 3(a) :Hysteresis with binary code 111

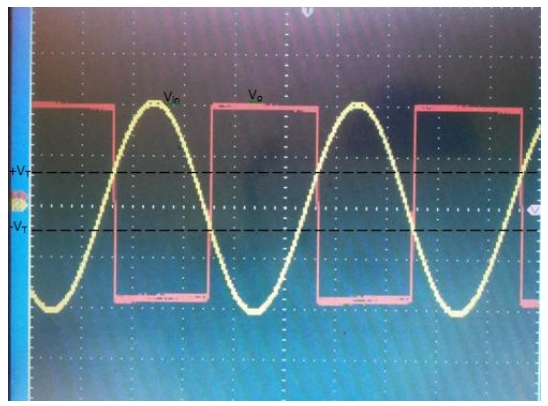

Fig.3(b): Hysteresis with binary code 100

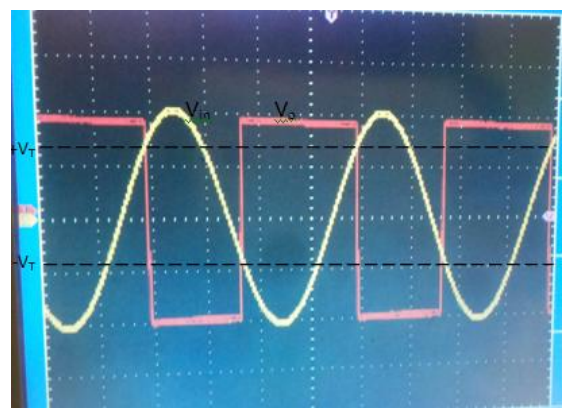

Fig. 3(c): Hysteresis with binary code 001 


\section{CONCLUSION}

A simple digitally tunable hysteresis Schmitt trigger with minimum hardware is presented. The circuit uses only one operational amplifier which makes it economical for low cost systems.

\section{REFERENCES}

[1] Fei Yuan, "A high speed differential CMOS Schmitt trigger with regenerative current feedback and adjustable hysteresis", Analog Integrated Circuits and Signal Processing, , Vol. 63, pp. 121-127, issue 1, 2010.

[2] SharamMinaei and EkramYuce, "A simple Schmitt trigger circuit with grounded passive elements and its applications to square/triangular wave generator", Circuits, systems and signal processing, Vol. 231, pp 877-888, June 2012.

[3] Marzaki A, Bidal V, Rahajandraibe W, Portal J L \&Bouchakour R, "New Schmitt trigger with controllable hysteresis using dual control gate-floating gate transistor (DCG-FGT)", International journal of reconfigurable and Embedded systems, Vol. 2, No.1, pp. 49-54, March 2013.

[4] Wuttipong Tamnupan and VarakornKasemsuwan, "A low voltage low power current mode differential adjustable Schmitt trigger", $9^{\text {th }}$ International conference on Electrical Engineering/Electronics, Computer, Telecommunication and information, ieeepp 1-4, 2012.
[5] Katyal Vipul, Geiger Randall L \& Chen Degang J, "Adjustable Hysteresis CMOS Schmitt Triggers", IEEE International Symposium on Circuits and systems, pp. 1938-1941, 2008

[6] Jiri Misurec and Jaroslav Koton, "Schimtt trigger with controllable hysteresis using current conveyors", International journal of advances in telecommunication, Electrotecnics, signals and systems, Vol. 1, No.1, pp 2630, 2012.

[7] W.Prodanov, M. C Schneider, "A digitally programmable current Schmitt-trigger. In: XVI International Conference on Microelectronics and Packaging,Pirenópolis - GO. SBMICRO 2001, pp. 106-110, 2001.

[8] Ramakant A. Gayakyard, "Operational Amplifiers and linear Integrated circuits", Prentice Hall of India $4^{\text {th }}$ Edition.

\section{AUTHOR PROFILE}

Faroze Ahmad received M.Sc. and Ph.D in Electronics in 2000 and 2007 respectively from University of Kashmir, Srinagar, India. He is presently serving as Assistant Professor in $\mathrm{E} \& \mathrm{C}$ Engineering at Islamic University of Science and Technology (IUST) Awantipora, J\&K India. The major areas of research include Message Encryption and Spread Spectrum Communication System. 